\title{
Abstracts
}

\section{General Care Issues Session 2}

\author{
The Swedish Myelomeningocele Follow-Up \\ Program (MMCUP) \\ Josenby-Lundkvist, Annika ${ }^{3}$, Alriksson-Schmidt, \\ $\mathrm{Ann}^{1,2}$, Westbom, Lena ${ }^{4}$ \\ ${ }^{1}$ Lund University, Dept. of Clinical Sciences, Orthope- \\ dics, Lund \\ ${ }^{2}$ Skåne University Hospital \\ ${ }^{3}$ Lund University Department of Health Sciences \\ ${ }^{4}$ Lund University, Department of Clinical Sciences \\ Lund, Paediatrics
}

Background Myelomeningocele (MMC) is a complex condition and individuals with MMC are at risk of severe medical complications and the development of secondary conditions. MMCUP - a Swedish multidisciplinary follow-up program and quality registry based on clinical evidence-based guidelines - began in 2012 with the goal to detect early signs of severe complications and to monitor changes over time. The purpose of this presentation is to describe the development and the current status of the program as well as its potential usefulness in health promotion and prevention.

Methods Data on a number of factors and domains are collected over time (body function, activities, participation, and treatment), and the data are entered into web-based questionnaires.

Results At the beginning of 2016, data on 142 children (born 2007-15) with a confirmed diagnosis of MMC had been entered into the MMCUP. Five of the children had died at a young age. Fifty percent of the children had undergone neurosurgery related to hydrocephalus. The prevalence rate of children with MMC born 2007-2015 and residing in Sweden according to MMCUP data was $1.44 / 10,000$ children $(95 \% \mathrm{CI}=$ $1.22-1.66 / 10,000)$. Fifty-three percent of these were boys.

Conclusions The development of the register has evolved over time and patients have been engaged in this work from the beginning. Professionals, as well as patients, are now eager to use the registry, and an increase of participants of all ages who want to partake in the registry has been noted. In September 2016, 365 participants ( $0-65$ years of age) were enrolled, and more than 3,000 assessment forms from different disciplines had been entered into MMCUP. Still, challenges related to the development of relevant and evidencebased data forms and questionnaires, and of getting the professionals involved to enter the data, exist.

\section{Clinic Passport to Improve Patient and Caregiver Satisfaction during a Multidisciplinary Clinic Rohr, Sarah E., O’Neil, Joseph Riley Hospital for Children}

Background During Spina Bifida Clinic appointments, patients see multiple providers from different disciplines. A common complaint from caregivers is the confusion of clinic flow. A passport system was developed, piloted, and implemented to improve patient and caregiver satisfaction. The passport is given families at the beginning of each appointment, marked with what services they will see. When a provider has completed the visit, they sign off by their service, to mark progress during the complex visit. When all boxes are signed, the visit is complete. This abstract summarizes the results of this quality improvement evaluation.

Methods The passport was designed to list which services were indicated for each child during the clinic visit, names of the providers, and service provided. An effectiveness and satisfaction survey was done for thirty days following the initiation of the passport system. After the visit, caregivers were given voluntary surveys using a 5 point Likert scale to assess their satisfaction with the passport and allowed open responses regarding clinic satisfaction. Descriptive statistics were used to present the data.

Results During the study period, 26 families piloted the passport and were asked to complete a voluntary survey. Twenty-two caregivers completed the survey 
and 17 of $22,(77 \%)$ found the passport helpful to very helpful, and 5 of 22, (23\%) of found the survey somewhat helpful. Spaces were provided for caregiver comments and were very positive.

Conclusions The passport system appears to improve satisfaction in clinic and the majority found it helpful, to very helpful. The largest barrier to success, was failure of providers to sign the form. Measures were taken to ensure passports were signed. Passports facilitate the ability for caregivers to understand clinic flow and improves patient and caregiver satisfaction and helps put them in control of their appointments.

Developing a community based service model: Making evidence informed decisions by listening to the voices of adults and children with Spina Bifida and hydrocephalus (SBH), their families and health professionals

Collins, Katrina

The Crann Centre

Background The purpose of this piece of research was to inform the strategic and operational development of the Crann Centre, Cork, Ireland, by engaging stakeholders, professional and nonprofessional, in a participatory evaluation process. These were contextualized within the current strategic and policy landscape of disability service provision in Ireland.

Methods A multi method approach was employed through the use of surveys, interviews and focus group methods. One hundred and twenty-one adults/children with $\mathrm{SBH}$ and their families took part in the research with an additional 37 health professionals participating in the process. The Framework Method was used to manage the voluminous nature of in depth interviews. The final framework consisted of 66 codes, clustered into 13 categories.

Results Describing the preferred service model for the Crann Centre emerged from a comprehensive and exhaustive analysis of responses $(n=151)$. Application of the Framework Method identified 16 thematic pillars. Summatively these pointed to Community based provision with the potential to be a centre of excellence for education, training and support across a number of levels;

- Tailored education programmes.

- Central coordinated information hub at a singular host point (multimedia).

- Social, Practical, Vocational and Transition support.

- Advocacy for patients and families.
- Professional development.

Conclusions Decision making around service model design at the Centre Centre was informed by the needs and expertise of persons with $\mathrm{SBH}$, their families and key informants from the statutory, voluntary and private sectors. The research has directly influenced plans to develop an innovative, responsive model of care driven by an ethos of inclusion, participation and empowerment that supports a social understanding of disability and holistic rehabilitation.

\section{Mobile Technology to Facilitate Health and Independence among Individuals with Disabilities: Overview of the University of Michigan Rehabilita- tion Engineering Research Center}

Meade, Michelle A.

University of Michigan

Background Adolescents and young adults with disabilities often show increased rates of secondary conditions as they make the transition from parental management of health to self-management and independence. While lack of knowledge, undeveloped skills, or poor choices can be strong contributors to these complications, impairments in cognition, including executive dysfunction, may also make it difficult to complete the tasks required to avoid them. Technology offers new opportunities to develop and implement strategies and interventions to support or reinforce specific, identified behaviors.

Methods The Technology Increasing Knowledge: Technology Optimizing Choice Rehabilitation Engineering and Research Center (TIKTOCRERC) is an interdisciplinary collaboration of clinicians and researchers from the School of Medicine, the College of Engineering, the College of Pharmacy, and the School of Information within the University of Michigan. The over-arching aim of the TIKTOC-RERC is to develop and evaluate innovative Rehabilitation Strategies, Techniques and Interventions that can be incorporated into healthcare practice and the lives of individuals with disabilities in order to enhance health, participation, and employment outcomes. Specifically, our Center focuses on optimizing decision-making and behaviors associated with health and independence among adolescents and young adults with physical, cognitive and neurodevelopmental disabilities.

Results The TIKTOC RERC supports 2 research and 4 development projects. A description of each project, and its current status, will be provided. 
Conclusions The TIKTOC RERC offers projects and products which can be used to promote the health and independence of adolescents and young adults with Spina Bifida and other disabilities.

Did you Look: Healthy Skin Starts with You. A Care Bundle to Promote Healthy Skin in People with Spina Bifida

Beierwaltes, Patricia S. ${ }^{1}$, Dicianno, Brad E. ${ }^{2}$, Smith, Kathryn $^{3,4}$, Wilhelmy, Jennifer ${ }^{5}$, Castillo, Jonathan ${ }^{6,7}$, Carey, Margaret ${ }^{8}$, Griffey, Judy ${ }^{8}$, Hobensack, Victoria $^{8}$, Ramen, Lisa ${ }^{9}$, Ong, Katherine ${ }^{10}$, Thibadeau, Judy $^{10}$

${ }^{1}$ Minnesota State University-Mankato

${ }^{2}$ University of Pittsburgh Medical Center

${ }^{3}$ Keck School of Medicine, University of Southern California

${ }^{4}$ Children's Hospital Los Angeles

${ }^{5}$ Gillette Children's and Lifetime Specialty Healthcare

${ }^{6}$ Baylor College of Medicine

${ }^{7}$ Texas Children's Hospitla

${ }^{8}$ Nationwide Children's Hospital

${ }^{9} \mathrm{SBA}$

${ }^{10} C D C$

Background The prevalence and cost of complications related to wounds in the Spina Bifdia population are well documented. Wound related issues are reported to be the second most common primary diagnosis in Spina Bifida admissions (Dicianno, B. \& Wilson, R., 2010). Kim et al. (2015) explored factors associated with pressure ulcers using data from the National Spina Bifida Patient Registry (NSBPR) and reported 26\% of these patients reported having had a pressure ulcer. The objective of this study was to develop, promote, and evaluate a care bundle that will impact skin integrity outcomes in people with Spina Bifida.

Methods The goal of the DYL bundle was to prevent a variety of skin breakdowns among individuals with Spina Bifida. It was modeled after the Agency for Healthcare Research and Quality guideline for preventing pressure ulcers. It has three integral parts: a skin breakdown risk assessment, skin assessment, and patient/family education. Ten clinics that are part of the National Spina Bifida Patient Registry (NSBPR) were trained on the bundle and are currently implementing it. The project is being monitored quarterly for continuous quality improvement and will be systematically evaluated using data from NSBPR.

Results Incidence rate of skin breakdown among participating clinics pre-implementation will be compared to post-implementation rate. Participating clinics' data will also be compared to non-participating clinics'. Though outcomes have not yet been analyzed, the skin care bundle is presented to promote attention to risk factors, skin assessment, and patient education.

Conclusions It is expected that the skin care bundle will improve outcomes for people with Spina Bifida of all ages.

\section{Impact of Motor Level and Spinal Curvature on Pulmonary Function in Spina Bifida}

Crytzer, Theresa M. ${ }^{1,2,3}$, Cheng, Yu-Ting ${ }^{1}$, Bryner, Mary $\mathrm{Jo}^{4}$, Wilson III, Robert ${ }^{4}$, Sciurba, Frank ${ }^{4}$, Dicianno, Brad E. ${ }^{1,2,3}$

${ }^{1}$ University of Pittsburgh

${ }^{2}$ University of Pittsburgh Medical Center, Center for Assistive Technology

${ }^{3}$ Human Engineering Research Laboratories

${ }^{4}$ University of Pittsburgh Emphysema/COPD Research Center

Background Pulmonary dysfunction in people with Spina Bifida (SB) is a common source of morbidity/mortality. We evaluated contribution of level of respiratory muscle denervation, scoliosis and obesity to pulmonary dysfunction.

Methods Spirometry and lung volumes were measured. Defined categories of impairment included restrictive lung disease, defined as total lung capacity (TLC) $<80 \%$ predicted with a forced expiratory volume in one second/forced vital capacity (FEV1/FVC) $>70 \%$; spirometric restriction as TLC $>80 \%$ predicted and $\mathrm{FVC}<80 \%$ predicted and obstructive lung disease as FEV1/FVC $<70 \%$.

Results Twenty-nine subjects (15 female; age $30 \pm$ 12 yrs.) participated. A significant negative association was found between motor level and FVC, FEV1, and TLC and between severity of scoliosis and FVC, FEV1 and TLC. Multivariate analysis showed significant negative association between motor level and FVC\%predicted (pred) $(p<0.0001)$, FEV1\%pred ( $p<0.0001)$, and TLC\%pred $(p<$ $0.0001)$. Severity of scoliosis was negatively associated with FEV1\%pred $(p=0.037)$ and TLC\%pred ( $p=0.047)$. Distribution of pulmonary function impairment category was $55 \%(n=16)$ restriction (low TLC), $6.9 \%(n=2)$ spirometric restriction, isolated decrease in FVC, $3.4 \%(n=1)$ combined obstruction and restriction, and $35.5 \%(n=10)$ normal. Restrictive pulmonary function was observed in $8 / 9(88 \%)$ 
people with thoracic level compared to lumbar level lesions.

Conclusions Pulmonary restriction was common. More rostral thoracic motor level was associated with higher degree of pulmonary dysfunction. Lung function indices (ie,TLC,FVC,FEV1) are impacted independently by motor level and scoliosis severity. Lumbar lesions which affect abdominal muscle expiratory efforts impacted ERV with preserved or increased RV impinging on FVC, whereas thoracic lesions resulting in defects of both intercostal inspiratory and abdominal muscle innervation impacted both IC as well as ERV and impacted on both TLC and FVC.

Fasting Serum Blood Measures of Bone and Lipid Metabolism in Children with Myelomeningocele for Early Detection of Cardiovascular and Bone Fragility Risk Factors

Van Speybroeck, Alexander ${ }^{1,2}$, Mueske, Nicole ${ }^{1}$, Mittelman, Steven ${ }^{1,2}$, Kremer, Richard ${ }^{3}$, Ryan, Deirdre $^{1,2}$, Wren, Tishya ${ }^{1,2}$

${ }^{1}$ Children's Hospital Los Angeles

${ }^{2}$ University of Southern California

${ }^{3}$ Mc Gill University Health Centre

Background Children with myelomeningocele (MM) may have an elevated risk for cardiovascular disease (CVD), metabolic syndrome, kidney disease, and bone fragility, early signs of which may be revealed in blood panels. This study examined serum levels in children with MM to determine whether pre-clinical signs of disease were present.

Methods Twenty-eight children with MM (93\% Hispanic; 17 males; $10.0 \pm 2.1$ years) and 58 typically developing children (84\% Hispanic; 30 males; $10.4 \pm$ 2.4 years) provided $>8$-hour fasting blood samples with concomitant dual-energy $\mathrm{x}$-ray absorptiometry measurements of body fat

Results Children with MM had higher body fat (35.2\% versus $29.9 \% ; p=0.01$ ) and altered lipid profiles (lower high-density lipoprotein levels, $43.9 \mathrm{mg} / \mathrm{dL}$ verses $51.6 \mathrm{mg} / \mathrm{dL}, p=0.03$ ) compared to typically developing children, suggesting elevated risk of metabolic syndrome. Children with MM also had significantly lower levels of calcium $(9.4 \mathrm{mg} / \mathrm{dL}$ verses $9.7 \mathrm{mg} / \mathrm{dL}$, $p=0.09)$, parathyroid hormone $(14.5 \mathrm{pg} / \mathrm{mL}$ verses $18.4 \mathrm{pg} / \mathrm{mL}, p=0.02)$ and alkaline phosphatase (187.0 U/L verses 237.0 U/L, $p=0.003)$, as well as a higher prevalence of vitamin D deficiency ( $43 \%$ versus $17 \%, p=0.02)$ suggestive of altered sensing mech- anisms of the parathyroid gland's response to normal physiological stimuli. After including trunk fat as a covariate, group differences persisted for creatinine $(p<$ $0.001)$, adjusted calcium ( $p=0.04)$, parathyroid hormone $(p=0.008)$ and vitamin $\mathrm{D}(p=0.009)$.

Conclusions Children with MM have abnormal biochemical markers for cardiovascular disease, insulin resistance and bone and mineral metabolism. Elevated CVD and metabolic syndrome risk factors are primarily associated with greater obesity, while the musculoskeletal risk factors may be reflective of MM. Early recognition and monitoring of these risk factors in patients with MM may help prevent later complications.

\section{Risk Factors for Advanced Skeletal Maturity in Children and Adolescents with Myelomeningocele Roiz, Ronald ${ }^{1}$, Mueske, Nicole ${ }^{1}$, Van Speybroeck, Alexander $^{1,2}$, Gilsanz, Vicente ${ }^{1,2}$, Ryan, Deirdre ${ }^{1,2}$, Wren, Tishya ${ }^{1,2}$ \\ ${ }^{1}$ Children's Hospital Los Angeles \\ ${ }^{2}$ University of Southern California}

Background Skeletal development is often affected in youth with myelomeningocele (MM). The underlying reasons have not been fully elucidated, though effects of mechanical loading, nutrition, bone metabolism, and the endocrine system have been investigated. This study assessed skeletal maturity, as measured by bone age, in children and adolescents with MM and examined the effects of sex, BMI, shunt status, and functional level.

Methods Fifty-two males (mean age: 10.1 years, range: 6.0-15.8 years; 10 sacral, 6 low lumbar, 28 mid lumbar, 8 high lumbar/thoracic) and 40 females (9.9 years, 6.2-16.9 years; 12 sacral, 7 low lumbar, 17 mid lumbar, 4 high lumbar/thoracic) with MM underwent $\mathrm{x}$-rays of their left wrists and hands for bone age determination. Bone age was evaluated separately for males and females. The difference between bone age and chronological age was assessed using a paired ttest. Other factors influencing bone age were evaluated using Spearman's Rho and multiple linear regression. Results Average bone age for both males and females was $10.7 \pm 3.9$ years. Bone age was significantly advanced compared to chronological age for both sexes (males: $0.64 \pm 1.6$ years advanced; females: $0.82 \pm$ 1.6 years; $p \leqslant 0.006$ ). Before age 9.5 years for males and 9.1 years for females, bone age was slightly delayed but then became advanced. No significant effects of neurosegmental level or shunt status were found. 
BMI was positively correlated with advanced bone age in both males $\left(r^{2}=-0.59, p<0.0001\right)$ and females $\left(r^{2}=-0.55, p<0.0001\right)$.

Conclusions Skeletal development is altered in children and adolescents with MM. Advanced bone age was observed in children with MM of both sexes after about 9 years of age. Skeletal maturity determination is important for timing of orthopaedic interventions common in this population, and a better understanding of bone age in children with MM may aid in surgical planning. 